\title{
Validation of a new adaptive deformable mirror concept
}

\author{
Roger Hamelinck ${ }^{a, c}$, Rogier Ellenbroek ${ }^{b}$, Nick Rosielle ${ }^{c}$, Maarten Steinbuch $^{a}$, Michel \\ Verhaegen $^{b}$ and Niek Doelman ${ }^{c}$ \\ ${ }^{a}$ Technische Universiteit Eindhoven, Den Dolech 2, $5600 \mathrm{MB}$, The Netherlands \\ ${ }^{b}$ Delft University of Technology, Mekelweg 2, 2628 CD Delft, The Netherlands \\ ${ }^{c}$ TNO Science and Industry, Stieltjesweg 1, 2628 CK Delft, The Netherlands
}

\begin{abstract}
A new prototype adaptive deformable mirror for future AO-systems is presented that consists of a thin continuous membrane on which push-pull actuators impose out-of-plane displacements. Each actuator has $\pm 10 \mu \mathrm{m}$ stroke, nanometer resolution and only mW's heat dissipation. The mirror's modular design makes the mechanics, electronics and control system extendable towards large numbers of actuators. Models of the mirror are derived that are validated using influence and transfer function measurements. First results of a prototype with 427 actuators are also presented.
\end{abstract}

Keywords: Deformable mirror, extendible, low power, modeling, validation, distributed control

\section{INTRODUCTION}

The Technische Universiteit Eindhoven, the Delft University of Technology and TNO Science and Industry have developed a prototype adaptive deformable mirror (DM) for future adaptive optics (AO)-systems. ${ }^{1,2}$ The mirror consists of a thin continuous membrane on which push-pull actuators impose out-of-plane displacements. The low voltage electromagnetic actuators are of the variable reluctance type and are located in an actuator plate. Each actuator has $\pm 10 \mu m$ stroke and nanometer resolution.

The modular design makes the mechanics and electronics extendable towards large numbers of actuators. The system is designed for a closed loop control bandwidth of $200 \mathrm{~Hz}$ and has a first resonance frequency above $1 \mathrm{kHz}$. The latter does not significantly decrease as the system is extended. ${ }^{2}$ Further, each actuator's heat dissipation is only mW's, avoiding the need for active cooling.

In this report a design overview of the entire DM system including the communication and driving electronics will be given (section 2). In section 3 linear time-invariant (LTI) system models are derived. These are used for detailed design decisions and will proof valuable tools for the design of a control system. They can straightforwardly be extended to larger DM designs with more actuators. In section 4 , besides the optical quality of the different DM's, the transfer function measurements of the actuators and influence function measurements are presented. These measurements show a high degree of linearity and correspond very well to the models.

In the very near future, the assembly of the large prototype that consists of seven modules with a total of 427 actuators will be completed. Promising test results will be presented in section 5 .

\section{DESIGN CONCEPT}

To keep actuator forces and heat dissipation low, a reflective continuous membrane is chosen as the correction element because of the low moving mass and low out-of-plane stiffness. Under this membrane a grid of electromagnetic low-voltage push-pull actuators is located. These variable reluctance actuators consist of a closed magnetic circuit in which a permanent magnet $(\mathrm{PM})$ provides static magnetic force on a ferromagnetic core in a membrane suspension. The force is influenced by a current through the coil that is situated around the magnet to provide movement of the core. Thin rods are glued between the actuators and the reflective mirror membrane and link their positions. The bending stiffness of the membrane yields a smooth reflective surface. The design is schematically shown in figure 1 .

Further author information: (Send correspondence to) R. Hamelinck, E-mail: r.f.m.m.hamelinck@tue.nl, Telephone: +31 40 2474580. R. Ellenbroek, E-mail: r.m.l.ellenbeork@tudelft.nl, Telephone: +31 152783371

\footnotetext{
Adaptive Optics Systems, edited by Norbert Hubin, Claire E. Max, Peter L. Wizinowich,

Proc. of SPIE Vol. 7015, 70150Q, (2008)

0277-786X/08/\$18 - doi: 10.1117/12.787755
}

Proc. of SPIE Vol. $701570150 Q-1$ 


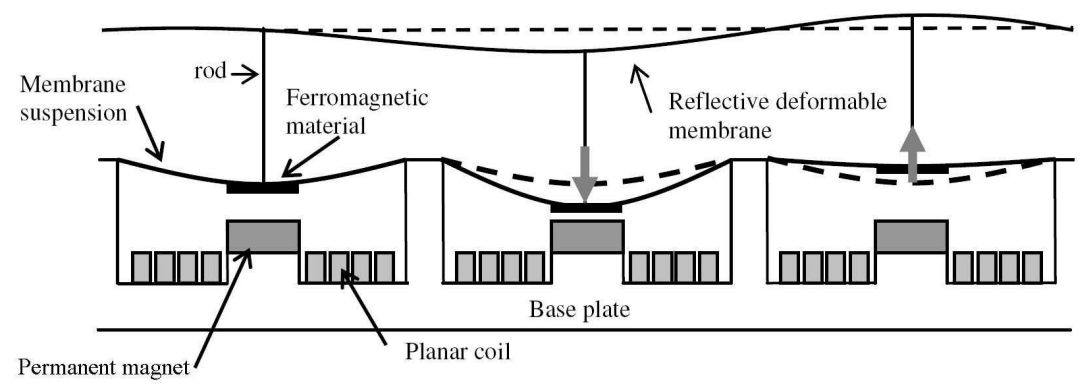

Figure 1. Schematic of the design. The variable reluctance actuators with the PM, coil, ferromagnetic core and suspension are shown. The actuators are coupled to the optical reflective membrane with rods.

\subsection{The actuators}

In this section the main actuator requirements, stiffness choice and consequences are briefly described.

Requirements. The actuator requirements that arise from the atmospheric conditions are derived in. ${ }^{1}$ The aim for an 8-meter wavefront in the visible $(550 \mathrm{~nm})$ is to decrease the root-mean-square (RMS) error in medium seeing conditions $\left.\left(r_{0}=0.166 \mathrm{~m}, \lambda=550 \mathrm{~nm}\right)\right)$ to $\frac{\lambda}{16}$. This results in the main specifications for the actuators:

- To minimize the fitting error $\left(\sigma_{f i t}^{2}=0.28\left(\frac{d}{r_{0}}\right)^{\frac{5}{3}}\right)$ the total number of actuators is 5000 , giving a $6 \mathrm{~mm}$ pitch for a $500 \mathrm{~mm}$ diameter mirror. This serves as a starting point in the design.

- To minimize the temporal error, $200 \mathrm{~Hz}$ control bandwidth is needed $\left(\sigma_{\text {temp }}^{2}=28.4\left(f_{G} \tau\right)^{\frac{5}{3}}, \tau=\frac{1}{2 \pi f_{\text {bandwidth }}}\right)$

- Total actuator stroke is set on $\pm 10 \mu \mathrm{m}$. This includes $5.6 \mu \mathrm{m}$ for the atmospheric disturbance and few additional $\mu \mathrm{m}$ 's to avoid saturation when small misalignments are present.

- The inter-actuator stroke is $0.4 \mu \mathrm{m}$.

- The resolution should be only a fraction of the wavelength and is set to $3 \mathrm{~nm}$.

Besides these requirements the actuators should not be subject to drift or hysteresis.

Stiffness. The actuator stiffness is an important parameter since it influences the mechanical resonance frequencies, the width of the influence function and the power dissipation. If the actuator stiffness is high the actuators will act as a hard point in the mirror surface and the first resonance frequency will be a waffle-like bending of the mirror, where the bending length will be equal to the actuator pitch. In this case, the frequency will not change with a mirror size increase and the mirror should be modeled as a plate.

On the other hand, if the actuators don't contribute to the stiffness of the mirror at all, the first resonance mode will be global bending of the mirror. Its frequency will depend on the very small out-of-plane stiffness of the mirror and will further decrease with a mirror diameter increase. The mirror is best modeled as a membrane and the pre-tension in the membrane will become important for the resonance frequency.

Both situations are not desired. Power dissipation in the stiff actuators is high, since the forces needed to move the actuators itself are high and if one actuator fails a hard point with a large negative optical effect will result. If the actuators are pure force actuators additional control stiffness is needed to control the internal modes of the mirror membrane. As a consequence local sensors and a local control loop are needed and significantly increase the overall complexity.

The actuator stiffness is chosen such that the first mechanical resonance frequency is high enough to allow for a closed loop bandwidth of $200 \mathrm{~Hz}$ without the need for additional local control loops. The mirror size is extendable without a change in resonance frequency, but the stiffness of the actuators should not be higher than needed to avoid excessive power dissipation and thereby avoiding the need for active cooling.

The actuator stiffness also influences the mechanical coupling and the influence function. The coupling $\eta$ is determined by: $\eta=\frac{c_{\text {mirror }}}{c_{\text {mirror }}+N^{\cdot c} c_{a c t}}$, where $c_{\text {mirror }}$ is the mirrors out-of-plane stiffness, $N$ is the number of adjacent actuators and $c_{a c t}$ is the actuator stiffness. A relative small coupling will keep the width of the influence function limited to one or two actuator rings and makes the system suitable for distributed control. 

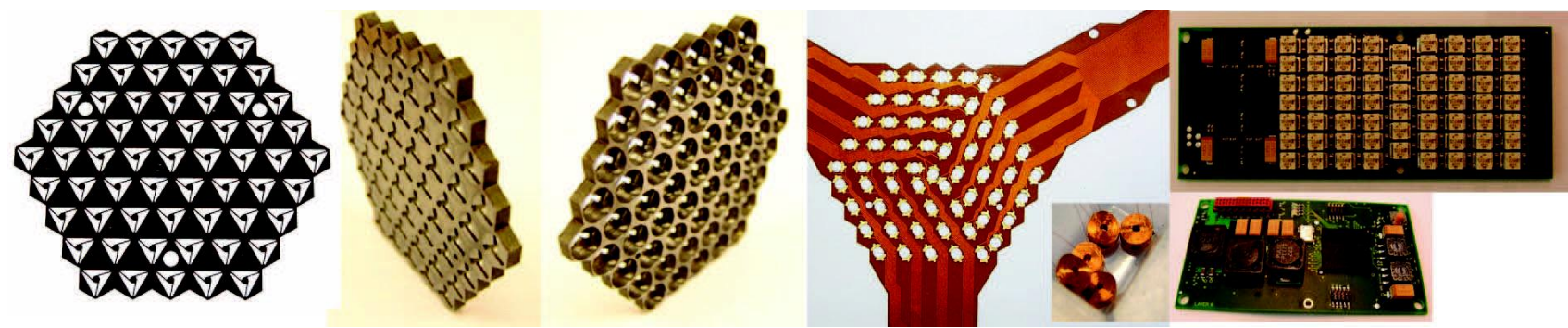

Figure 2. The manufactured components, from left to right: the $25 \mu \mathrm{m}$ thick membrane suspension, the baseplate, the flex foil to connect the coils to the PCB, the coils and the PCB with the driver electronics.

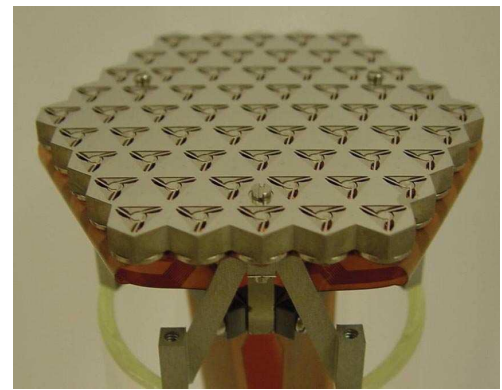

Figure 3. The topview of the actuator grid

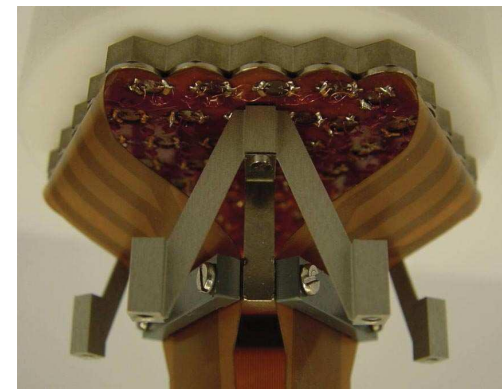

Figure 4. The actuator grid as seen from the bottom.

Design. The design of the variable reluctance actuator is described in. ${ }^{1}$ The goal is to realize efficient and low stiffness actuators that can be manufactured in arrays instead of separate and individually positioned actuators. The arrays are realized by stacking different layers. The main layers are shown in figure 2. On the left the $25 \mu \mathrm{m}$ thick high-strength membrane suspension for the ferromagnetic moving cores is shown. In the center, the front and backside of the baseplate, is shown. In the baseplate subassemblies with the permanent magnet (SmCo) and coil (500 windings, $50 \mu \mathrm{m} \mathrm{Cu}$-wire) are placed. The flex foil that connects the coil wires and the printed circuit board (PCB) with the driver electronics is shown on the right. The tile with 61 actuators serves as a standard building block for large arrays ${ }^{3}$ and is shown in figure 3 and figure 4.

\subsection{The membrane}

A continuous membrane is chosen as the correcting element because of its low moving mass and low out-of-plane stiffness. A continuous membrane gives the best correction for a given number of actuators. ${ }^{4}$ The low out-ofplane stiffness gives, together with the actuator stiffness, a local influence function given good opportunity for a distributed control strategy. ${ }^{2}$

There's a minimum thickness for the mirror membrane for a given material and a given maximum gravitational sag between the actuators. To minimize forces and power dissipation the thickness should not be more than the thickness set by this deflection limit. As shown in ${ }^{1}$ this thickness is $\sim 50 \mu \mathrm{m}$ for Beryllium and $\sim 100 \mu \mathrm{m}$ for glass with the $6 \mathrm{~mm}$ actuator pitch. For practical reasons, such as cost and availability, Pyrex mirrors with $100 \mu \mathrm{m}$ thickness from LightMachinery with a single sided $\mathrm{Al}$ and $\mathrm{SiO}_{2}$ coating were obtained and used for initial testing. The membranes where obtained in 2" and 6" diameter.

\subsection{Membrane actuator connection}

Each actuator imposes a single degree-of-freedom (DOF) in the mirror surface. The bending stiffness of the mirror will form a smooth surface through these out-of-plane displacements. Rods are used to transfer only a single DOF from the actuator to the mirror surface. As shown in figure 5 the bending stiffness of the rods is lower than that of the mirror, avoiding piston-like patterns in the surface and the resulting higher order abberations. This connection also leaves the mirror free to in-plane expansion and shrinkage with ambient temperature variations and variations between actuator modules. The length/diameter ratio of the rod is chosen such that $k_{\phi, \varphi}$ is smaller 


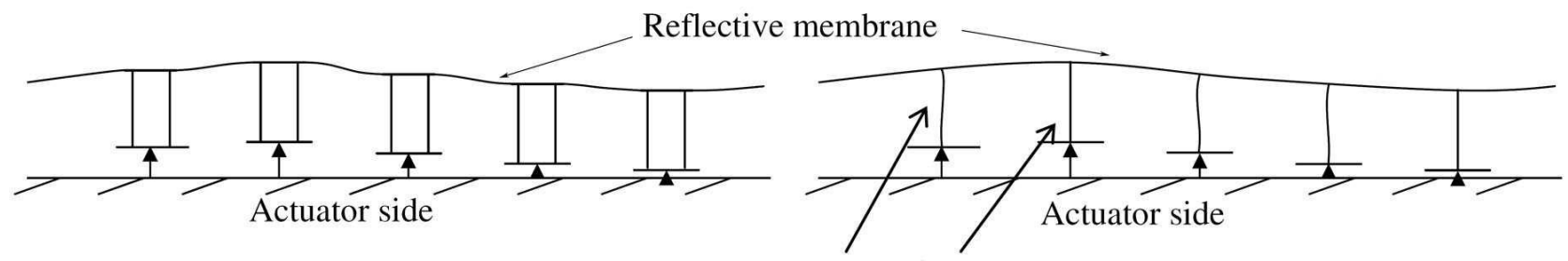

Thin rods

Figure 5. Actuators with high bending stiffness connected to the mirror surface constrain local tip/tilt which result in higher order abberation imposed in the reflecting wavefront. (left) With proper stiffness decoupling, e.g. by rods, a smooth mirror surface is formed. (right)

than that of the mirror at the connection and buckling and the rods violin modes are avoided. With small epoxy glue droplets the connection between the rods, the mirror membrane and the actuators is established. Since the forces on the connections are very small, the droplets needed can be very small as well $\left(\sim 10^{-11} \mathrm{~m}^{3}\right)$. In section 4.1 it will be shown that the decrease in optical quality as a result of the small droplets and the low-shrinkage of the glue is acceptable. Since the actuators only constrain the out-of-plane DOF's additional constraints are needed for the in-plane DOF's. Three folded leaf springs, tangentially placed at the circumference, constrain X, $\mathrm{Y}$ and $\theta$. These folded leafsprings are visible in figure 14 .

\subsection{Assembly}

Assembly of the mirror, rods and actuators is not trivial, but well possible with some additional tooling. Figure 6 shows how in a few steps the mirror and rods are connected to a dummy that represents the actuator modules. Since the mirror is very flexible a reference surface is used to enforce the membranes shape. A porous air bearing connected to a vacuum pump is used to 'suck' the membrane onto the reference surface of the air bearing. This vacuum pressure is maintained during the whole assembly process. In a second step small epoxy droplets are placed on the backside of the mirror. With the aid of a mould the rods are placed in the droplets and placed perpendicular to the mirror surface. When the epoxy is cured the mould is removed and a grid of rods remains. This is shown on the lower left part of figure 6. A identical grid of droplets is placed on the actuator grids, or in this illustrated case, on a rigid dummy. In the final step the mirror with the rods is placed on top of the dummy with the droplets.

In figure 4.1 it is shown that with this procedure a 6 " mirror can be attached to a stiff dummy with an initial flatness error $<2 \mu \mathrm{m}$ PTV. In figure 7 a detail of the glued connection is shown.

\subsection{Electronics}

Driver electronics. A comparison has been made between the power efficiencies of several designs for the driver electronics, leading to the choice for a class D or pulse-width modulation (PWM) amplifier. The output voltage of this amplifier is applied to the actuator, resulting in a current. Positive and negative currents are generated using an H-bridge layout (figure 8) in which four switches control the current path. The switches are driven by a PWM generator that uses BD modulation to provide switching signals A and B. This method has a lower power dissipation than traditional modulation methods and only half the voltage swing and thus ripple on the actuator position due to the non-stationary PWM voltage. This ripple is related to the system dynamics and the PWM base frequency $f_{p w m}$ chosen in the next section. The base frequency is strongly related to the power consumption of the FPGA used for implementation and must be minimized. Therefore a $2^{\text {nd }}$ order analog low-pass filter is applied and the 16-bit PWM is split into a course 11-bit PWM providing A and B and a fine 5-bit PWM providing C (figure 8).

Communication. The FPGA that generates the PWM signals also handles the serial communication connection with the control computer. To minimize power consumption at the receiving ends and maximize design freedom, a multi-drop, low-voltage differential signalling (LVDS) connection has been chosen. This full duplex line operates at $40 \mathrm{Mbit} / \mathrm{s}$ and can simultaneously drive up to 32 actuator modules. The protocol overhead is 

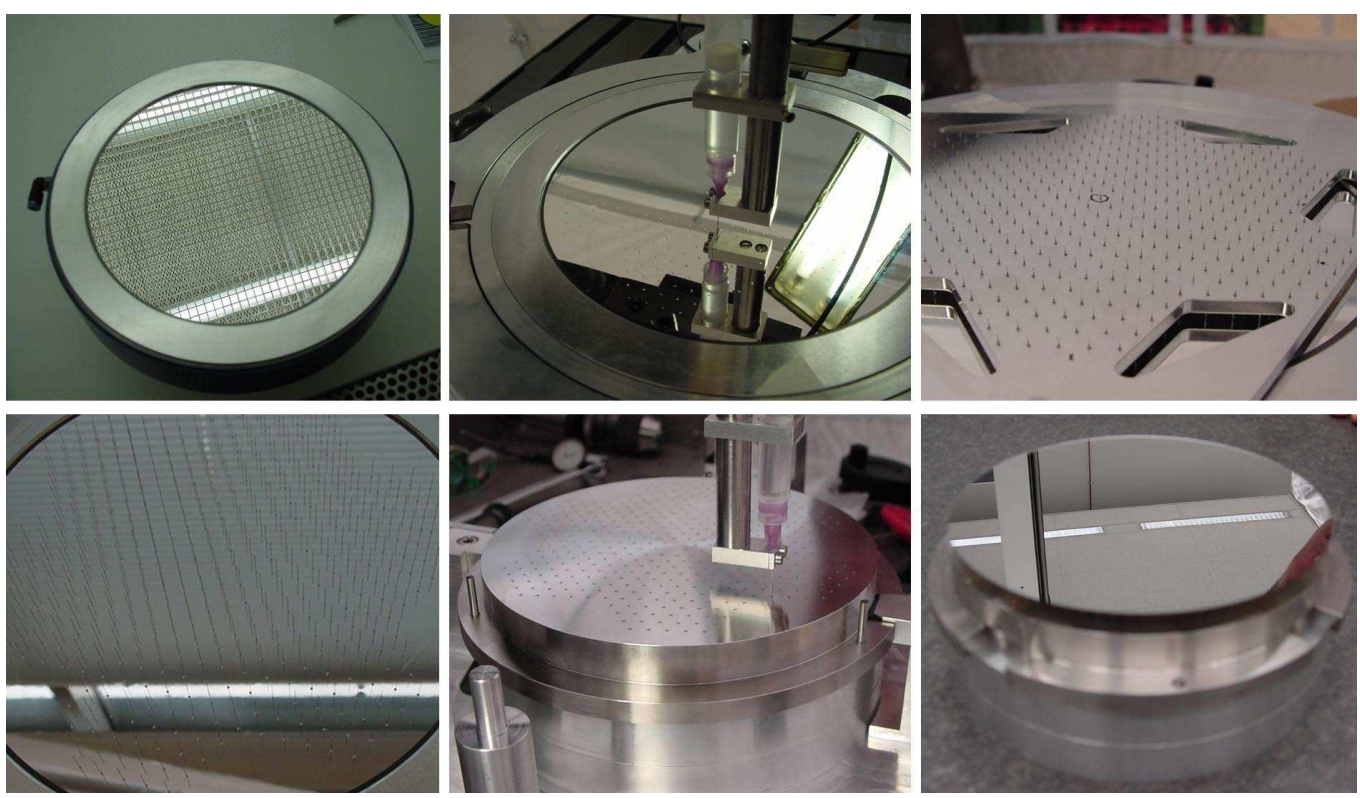

Figure 6. The rod placement process as described in section 2.4.
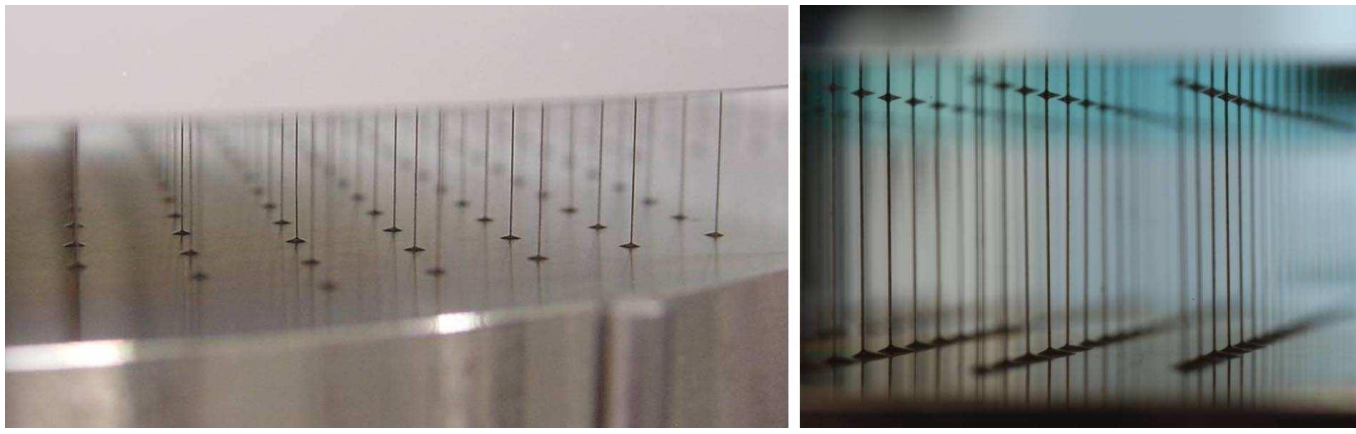

Figure 7. Details of the glued connection of the rods to the mirror and dummy.

minimal and only $30 \mu$ s is required to update one 61 actuator module. However, the actuators receive their setpoints sequentially such that the latency will have a slight, deterministic variation for each actuator. The return line is used for testing and monitoring functions to read back the setpoints or the results of the test for the integrity of the actuator coils.

In the final design this communication link to the control computer will be direct, but for the prototyping stage a communication bridge was developed that translates ethernet into LVDS and vice versa. This allows any commercially available computer to control the DM. For the ethernet communication the lean user datagram protocol (UDP) standard is used.

\section{MODELING}

\subsection{The actuator}

With an electrical equivalent circuit analysis the electromagnetic actuator is modeled. In the actuator force equilibrium exists between the (electro)magnetic force from the permanent magnet and coil and the restoring force from the mechanical spring in which the ferromagnetic core is suspended. This force is given by: $F_{m a g}=$ $\frac{-1}{2 \mu_{0} A_{m}}\left(\left(N I+H_{c} d_{m}\right) / R\right)^{2}$, in which $\mu_{0}$ is the permeability of vacuum, $A_{m}$ the magnet area, $N I$ the number of ampere-turns of the coil, $H_{c}$ the coercive force of the permanent magnet, $d_{m}$ the thickness of the magnet and $R$ the total reluctance of the magnetic circuit. The non-linear spring force is modeled as: $F_{s}=A \frac{E t^{3}}{r^{2}} z+B \frac{E t}{r^{2}} z^{3}$, in 


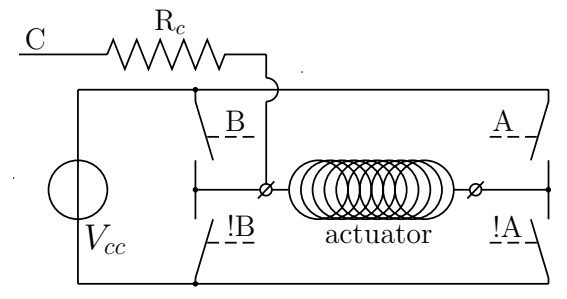

Figure 8. H-bridge layout for the PWM amplifier. The '!' means 'complementary'.

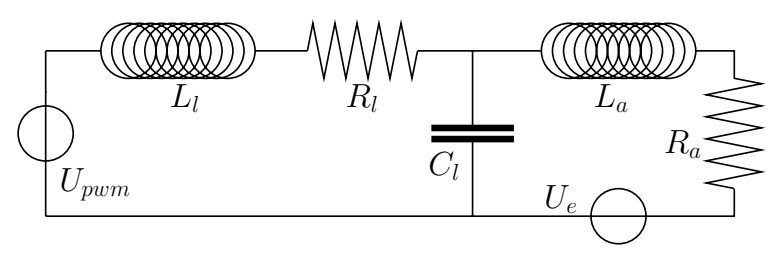

Figure 9. The analog electronic circuit.

which $E$ is the membranes Young's modulus, $t$ the membrane thickness, $r$ the radius of the suspension and $A$ and $B$ constants depending on the shape of the suspension. The stiffness in the actuation direction $z$ is the sum of the negative stiffness from the magnetic circuit and the larger positive stiffness from the membrane suspension.

\subsection{The membrane}

Since the actuator rods are only $100 \mu m$ thick, the force they exert on the reflective membrane can be considered to be a point-force. The membrane will be modeled as a thin plate because its bending moment can not be neglected. Let $z_{i}$ and $\zeta_{j}$ for $i=1 \ldots N_{z}$ and $j=1 \ldots N_{a}$ be complex values corresponding to coordinates in the complex plane. The deflection $h\left(z_{i}\right)$ of a circular, thin plate with radius $r_{r m}$ and free edge conditions due to a point force at $\zeta_{j}$ can be derived from the well known biharmonic plate equation ${ }^{5}$ in terms of the Laplacian operator $\nabla^{2}(z)$ and the plate's flexural rigidity $R_{r m}$ as:

$$
\nabla^{4} h(z)=\frac{f_{j}}{R_{r m}}, \text { where } \quad \nabla^{2}(z)=\frac{\delta^{2}}{\delta x^{2}}+\frac{\delta^{2}}{\delta y^{2}} \quad \text { and } \quad R_{r m}=\frac{E_{r m} h_{r m}^{3}}{12\left(1-\mu_{r m}^{2}\right) r_{r m}^{2}},
$$

$E_{r m}$ is the plate's Young's modulus, $\mu_{r m}$ its Poisson ratio and $h_{r m}$ its thickness. For a linear material model the deflection $h\left(z_{i}\right)$ can be expressed analytically in terms of $f_{j}$ as: ${ }^{4}$

$$
h\left(z_{i}\right)=\frac{f_{j}}{16 \pi R_{r m}} W\left(z_{i}, \zeta_{j}\right)+W_{p}+W_{x} \Re\left(z_{i}\right)+W_{y} \Im\left(z_{j}\right),
$$

where $W_{p}, W_{x}$ and $W_{y}$ denote the rigid body motions in the out-of-plane direction and around the $x$ - and $y$ axes respectively. Further, $\Re(z)$ and $\Im(z)$ denote the real and imaginary parts of $z$ respectively and

$$
\begin{aligned}
W\left(z_{i}, \zeta_{j}\right)= & \left(z_{i}-\zeta_{j}\right)\left(z_{i}^{*}-\zeta_{j}^{*}\right)\left\{\ln \left(z_{i}-\zeta_{j}\right)+\ln \left(z_{i}^{*}-\zeta_{j}^{*}\right)+\frac{1-\mu}{3+\mu}\left[\ln \left(1-z_{i} \zeta_{j}^{*}\right)+\ln \left(1-z_{i}^{*} \zeta_{j}\right)\right]\right\} \\
& +\frac{(1-\mu)^{2}}{(1+\mu)(3+\mu)} z_{i} z_{i}^{*} \zeta_{j} \zeta_{j}^{*} \\
& +\frac{8(1+\mu)}{(1-\mu)(3+\mu)}\left\{\left(1-z_{i} \zeta_{j}^{*}\right) \ln \left(1-z_{i} \zeta_{j}^{*}\right)+k\left(z_{i} \zeta_{j}^{*}\right)+\left(1-z_{i}^{*} \zeta_{j}\right) \ln \left(1-z_{i}^{*} \zeta_{j}\right)+k\left(z_{i}^{*} \zeta_{j}\right)\right\}, \\
k(x)= & \int_{0}^{x} \frac{\ln (1-\alpha)}{\alpha} d \alpha=-\operatorname{dilog}(1-x),
\end{aligned}
$$

where ${ }^{*}$ denotes the complex conjugate. This analytic expression allows the spatial grids to be discretized without loss of accuracy. Linearity is assumed to express the deflection due to multiple point forces using linear superposition.

Let $H_{z, i}=h\left(z_{i}\right)$ and $F_{\zeta, j}=f_{j}$ be elements of the vectors $H_{z}$ and $F_{\zeta}$. Similarly, the coordinates $z_{i}$ and $\zeta_{j}$ form the $i$ and $j^{t h}$ elements of the vectors $Z$ and $\bar{\zeta}$ and $\Omega_{z \zeta, i, j}=W\left(z_{i}, \zeta_{j}\right)$ the elements of the matrix $\Omega_{z \zeta}$. The mirror deflection $H_{z}$ can then be expressed as:

$$
H_{z}=\Omega_{z \zeta} F_{\zeta}+U_{z} M
$$



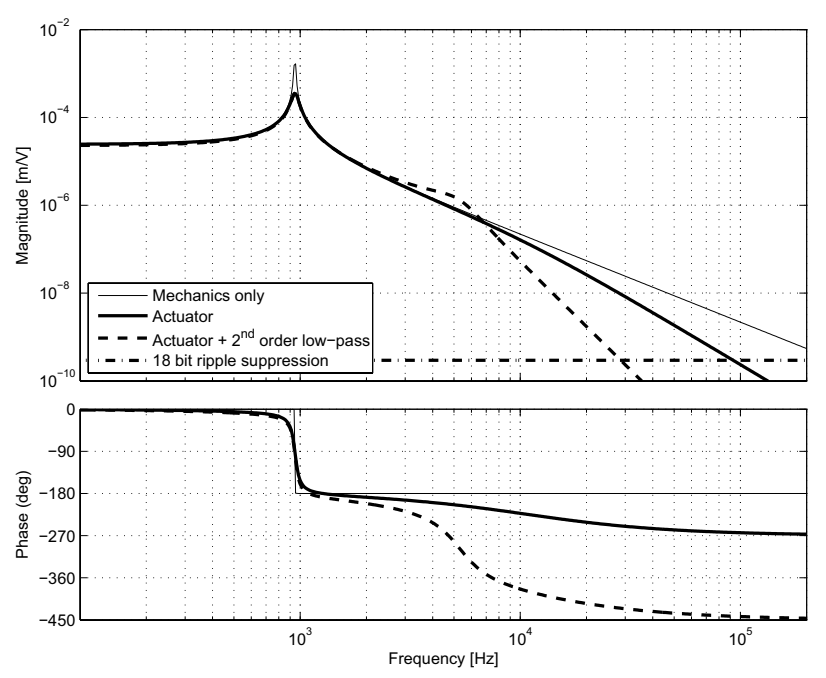

Figure 10. Bode diagram of the transfer functions between $U_{\text {pwm }}(t)$ and $h(t)$ for three different cases: only the mechanics, mechanics including the actuator coil and mechanics with actuator coil and $2^{\text {nd }}$ order low-pass.
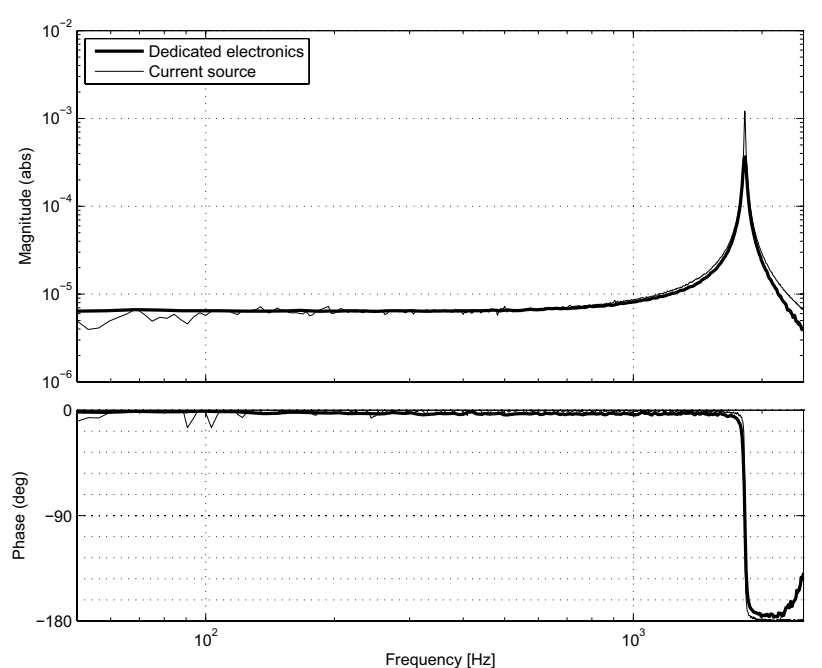

Figure 11. Bode plots of the measured transfer functions of a single actuator driven by a current source and the dedicated PWM voltage source. The first is scaled with the resistance $R_{a}$ for better comparison.

where $U_{z}=\left[\mathbf{1}^{N_{z} \times 1} \Re(Z) \Im(\bar{z})\right]$ and $M=\left[W_{p} W_{x} W_{y}\right]^{T}$. Since the rigid body modes are not constrained by the edge conditions of the plate, both the moments around the $\mathrm{x}$ and $\mathrm{y}$ axes due to the plate forces $F_{\zeta}$ as well as the mean plate force in the out-of-plane direction should be zero. This leads to an extra condition $U_{\zeta}^{T} F_{\zeta}=0$ that together with (1) evaluated only on the actuator coordinates $\bar{\zeta}$ can be expressed in matrix form. The relation can then be inverted to yield:

$$
\left[\begin{array}{c}
F_{\zeta} \\
M
\end{array}\right]=\left[\begin{array}{cc}
\Omega_{\zeta \zeta} & U_{\zeta} \\
U_{\zeta}^{T} & 0
\end{array}\right]^{-1}\left[\begin{array}{c}
H_{\zeta} \\
0
\end{array}\right]=\left[\begin{array}{c}
K_{m} \\
K_{r}
\end{array}\right] H_{\zeta}
$$

where the stiffness matrices $K_{m}$ and $K_{r}$ are implicitly defined.

\subsection{Influence functions}

The reflective membrane is supported by actuators with a linear mechanical stiffness, for which the following force equilibrium must be satisfied:

$$
F_{a}-C_{a} H_{\zeta}-F_{\zeta}=0
$$

where $C_{a}$ is a diagonal matrix whose $i^{\text {th }}$ diagonal element is the effective mechanical stiffness of actuator $i$ and the vector $H_{\zeta}$ contains the plate deflections at the actuator locations. Substitution of (2) for $F_{\zeta}$ into the force equilibrium in (3) yields $F_{a}-C_{a} H_{\zeta}-K_{m} H_{\zeta}=0$ and thus the mirror deflection $H_{\zeta}$ at the actuator positions is related to the actuator forces $F_{a}$ via the influence matrix $B_{\zeta}$ as:

$$
H_{\zeta}=B_{\zeta} F_{a} \quad \text { where } \quad B_{\zeta}=\left(K_{m}+C_{a}\right)^{-1}
$$

The plate deflections can also be evaluated over the arbitrary grid with coordinates $Z$. The results from (2) can be substituted into the plate equation in (1) together with (4), yielding $H_{z}=B_{z} F_{a}$ where $B_{z}=\left(\Omega_{z \zeta} K_{m}+U_{z} K_{r}\right) B_{\zeta}$. Two influence functions are plotted in figure 17.

\subsection{The electronics}

The H-bridge type PWM generator in figure 8 generates a voltage $U_{\text {pwm }}$ over the depicted actuator clamps. The electronic circuit of the actuator system connected to these clamps is depicted in figure 9 . The actuator coil is denoted $L_{a}$ and has electrical resistance $R_{a}$. Via the motor constant $K_{t}$, a current through this coil yields a proportional mechanical actuator force $F_{a}$. Variation of the actuator position $h$ then generates a back-emf 
Table 1. Estimated and designed values of actuator and electronics properties

\begin{tabular}{c|c|c|c|c|c|c|c|c|c} 
Parameter & $L_{l}$ & $R_{l}$ & $C_{l}$ & $L_{a}$ & $R_{a}$ & $K_{t}$ & $m_{a}$ & $b_{a}$ & $c_{a}$ \\
\hline Value & 220 & 2.7 & 4.7 & 571 & 39 & 0.48 & 7.5 & $10^{-5}$ & 500 \\
Unit & $\mu \mathrm{H}$ & $\Omega$ & $\mu \mathrm{F}$ & $\mu \mathrm{H}$ & $\Omega$ & $\mathrm{N} / \mathrm{A}$ & $\mu \mathrm{g}$ & $\mathrm{Ns} / \mathrm{m}$ & $\mathrm{N} / \mathrm{m}$
\end{tabular}

voltage, denoted by $U_{e}$. The second order analog low-pass filter consists of coil $L_{l}$ with internal resistance $R_{l}$ in series with capacitor $C_{l}$. Using Kirchoff's laws and the corresponding constitutional equations, the electronic circuit can be modeled in a state-space form as:

$$
\left[\begin{array}{c}
\dot{I}_{R_{l}} \\
\dot{I}_{R_{a}} \\
\dot{U}_{C_{l}} \\
\dot{h} \\
\ddot{h}
\end{array}\right]=\left[\begin{array}{ccccc}
-R_{l} / L_{l} & 0 & -1 / L_{l} & 0 & 0 \\
0 & -R_{a} / L_{a} & 1 / L_{a} & 0 & -K_{t} / L_{a} \\
1 / C_{l} & -1 / C_{l} & 0 & 0 & 0 \\
0 & 0 & 0 & 0 & 1 \\
0 & K_{t} / m_{a} & 0 & -k / m_{a} & -b_{a} / m_{a}
\end{array}\right]\left[\begin{array}{c}
I_{R_{l}} \\
I_{R_{a}} \\
U_{C_{l}} \\
h \\
\dot{h}
\end{array}\right]+\left[\begin{array}{c}
1 / L_{l} \\
0 \\
0 \\
0 \\
0
\end{array}\right] U_{p w m} .
$$

The output signals that will be used for analysis and testing are the actuator displacement $h$ and the voltage $U_{C_{l}}$ that can be measured over actuator coil and are both present in the state. When the properties of the actuator and the electronics are taken as in table 1, then the transfer function in figure 10 is obtained.

The spectrum of the modulated PWM signal for a constant duty cycle $d \in[-1,1]$ contains energy at discrete frequencies $f=n f_{p w m}$ for $n \in \mathbb{N}$, of which only the DC component is of interest. The worst-case magnitude of these harmonics is found for $d=0.5 / n$ as $V_{c c} /(n \pi)$. Since the actuator system has a low-pass characteristic and the magnitude of the harmonic decreases with frequency, the first harmonic causes the most significant ripple on the actuator position. The ripple magnitude will be designed to be less than $1 / 4$ of the actuator displacement due to the least significant bit, corresponding to $\left|H\left(2 \pi j f_{p w m}\right)\right| 4 \cdot 2^{16}<\pi|H(0)|$, where $H(2 \pi j f)$ is the frequency response of the system.

In figure 10 the system response to the PWM voltage is plotted, together with the desired attenuation level. The system's frequency response is also plotted for the case that the $2^{\text {nd }}$ order low-pass filter is not present. This can be derived using the approach in the previous subsection. The filter reduces the required base frequency $f_{p w m}$ from above $100 \mathrm{kHz}$ to less than $30 \mathrm{kHz}$.

The clock frequency of the FPGA used for implementation must be $f_{f p g a}=2^{N_{b}} f_{p w m}$, where $N_{b}$ is the number of bits of precision of the PWM signal. For $N_{b}=16$ and $f_{p w m}=10^{5}$ this clock frequency is not feasible for current FPGA's. This and also the power dissipation is significantly reduced using the $2^{\text {nd }}$ order low-pass filter and the splitting of the PWM into a coarse and a fine part.

The goal is to replace the glass mirror membrane by beryllium, which has a higher specific stiffness that allows a thinner membrane and thus less mass per actuator. This increases the system's eigenfrequency and decreases the attenuation of the PWM ripple. Therefore, $f_{p w m}$ has been chosen at $61 \mathrm{kHz}$. For the foreseen sampling frequency of $1 \mathrm{kHz}$ this provides 61 times oversampling such that any cross-harmonics due to the non-constant setpoint signal can be neglected.

\subsection{Modeling for control}

Observe in figure 10 that the lowly damped mechanical resonance frequency of the actuator lies above the foreseen sampling frequency of $1 \mathrm{kHz}$. The consequences for closed loop control of a single, isolated actuator will now be analyzed using the aliased frequency response function (AFRF) concept proposed in. ${ }^{6,7}$ Let the continuous time plant $P(s)$ be described by (5) and its output $h(t)$ be measured using a sensor that integrates $h(t)$ over an exposure time $t_{e}$, similar to CCD-based Shack-Hartmann sensor. Its measurement is expressed in the Laplace domain as $y(s)=G(s) h(s),{ }^{8}$ where $G(s)=\frac{1-e^{-s t_{e}}}{s t_{e}}$. This signal is sampled with sampling frequency $f_{s}$ and input to the controller $C(z)$ that sends its output setpoint over the communication link with latency $\tau_{c}$ to the plant, while considering a zero-order hold $(\mathrm{ZOH})$. The communication latency is expressed as $e^{-s \tau_{c}}$ and the $\mathrm{ZOH}$ as $\frac{1-e^{-s T_{s}}}{s} .9$

The control loop thus contains both discrete time and continuous time components and the loop gain can be 

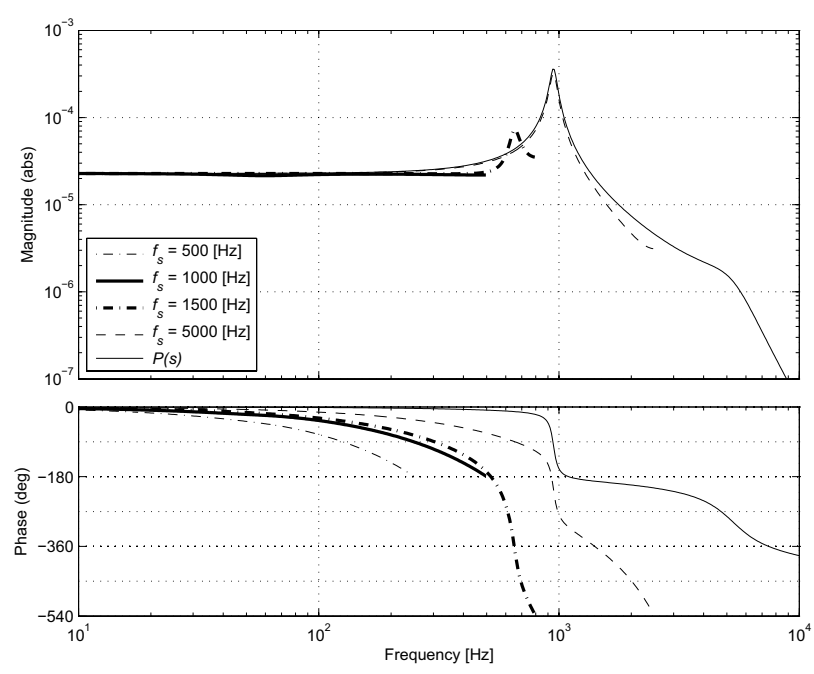

Figure 12. The AFRF of the single actuator system for various sampling frequencies when assuming $\tau_{c}=120 \mu \mathrm{s}$.

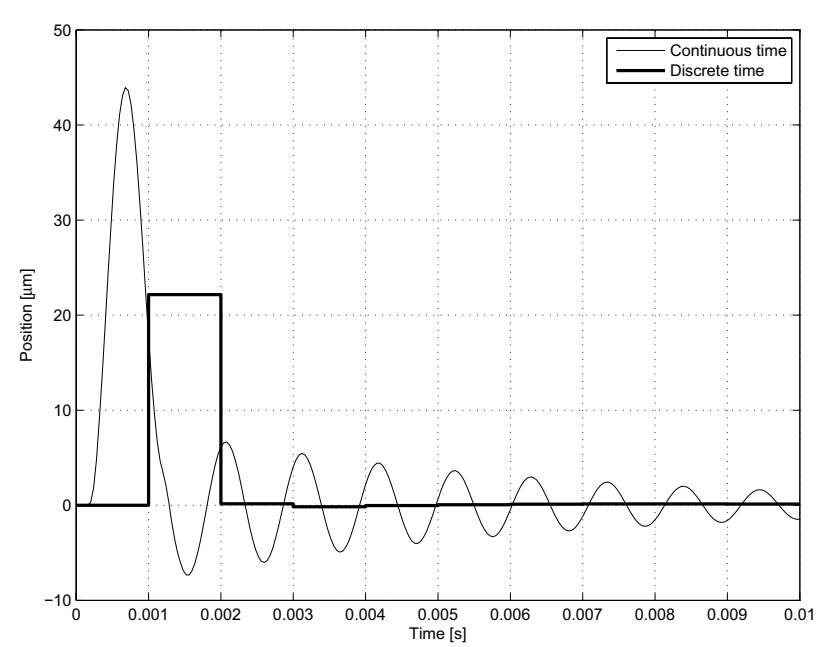

Figure 13. Impulse responses of the single actuator system for both the actual and discrete time measured position (AFRF) for $f_{s}=1 \mathrm{kHz}$.

written as $L(s, z)=G(s) P(s) \frac{1-e^{-s T_{s}}}{s} e^{-s \tau_{c}} C(z)$. This will be converted to a discrete time $\operatorname{AFRF} \tilde{L}(z)$ that models both the continuous time components and the aliasing effects introduced by sampling. ${ }^{6}$ This $\tilde{L}(z)$ can be obtained using the $z$-transform ${ }^{9}$ denoted by the operator $\mathcal{Z}$ as $\tilde{L}(z)=\mathcal{Z}(L(s, z))$. When assuming that $t_{e}=T_{s}$ and using $\mathcal{Z}\left(1-e^{-s T_{s}}\right)=1-z^{-1}$, then the remaining transformation can be performed using matlab's C2D command in which also the effect of the arbitrary time-delay $\tau_{c}$ can be accounted for. In figure 12 the AFRF's are plotted for $\tau_{c}=130 \mu \mathrm{s}$ and various sampling frequencies $f_{s}$. Observe that $f_{s}$ determines the frequency and magnitude of the aliased resonance frequencies, which for e.g. $f_{s}=1500 \mathrm{~Hz}$ will significantly reduce the achievable control bandwidth if the controller is restricted to an integrator structure.

The continuous time response of the actuator position to a discrete time impulse on $U_{p w m}$ is plotted in figure 13 together with the discrete time response corresponding to the AFRF. Observe the low damping of the single actuator system and the single sample of the discrete time response that has a significant magnitude. The latter suggests that a first or second order finite impulse response (FIR) model will be suitable for control synthesis. Finally, note that the model can be extended to the full DM system, leading to a similar discrete time response and a similar model structure for controller design.

\section{VALIDATION AND MEASUREMENTS}

After testing the optical quality of the new DM using the dummy mirror, this section will present validation measurements for the models derived in the previous section on the recently assembled prototype. This consists of a single module with 61 actuators (figure 14), one custom designed driver electronics module and the ethernetto-LVDS communications bridge.

\subsection{Optical quality}

The optical quality of the prototype DM was tested by gluing 2" and 6" mirrors with hexagonal grids of 61 and 427 rods to a dummy base. The dummy bases represent the actuator modules on which the membrane and rods will be glued in further prototypes. With an interferometer the optical quality is measured. The left interferograms in figures 15 and 16 show the shapes of the mirrors over the areas supported by rods. They are $\sim 3 \mu \mathrm{m}$ and $\sim 4.5 \mu \mathrm{m}$ PTV unflat, which - in theory - can be corrected using the actuators. The right images in figures 15 and 16 show the non-flatness after flattening, corresponding to $18 \mathrm{~nm}$ and $28 \mathrm{~nm}$ RMS respectively. To calculate the required actuator signals, note that the actual DM will be supported by soft actuators that will deflect and partly relax the measured non-flatness. For both the 61 and 427 rod dummies, all required actuator voltages are below $10 \%$ of the available force and correspond to $\sim 0.1 \mathrm{~V}$ RMS. 

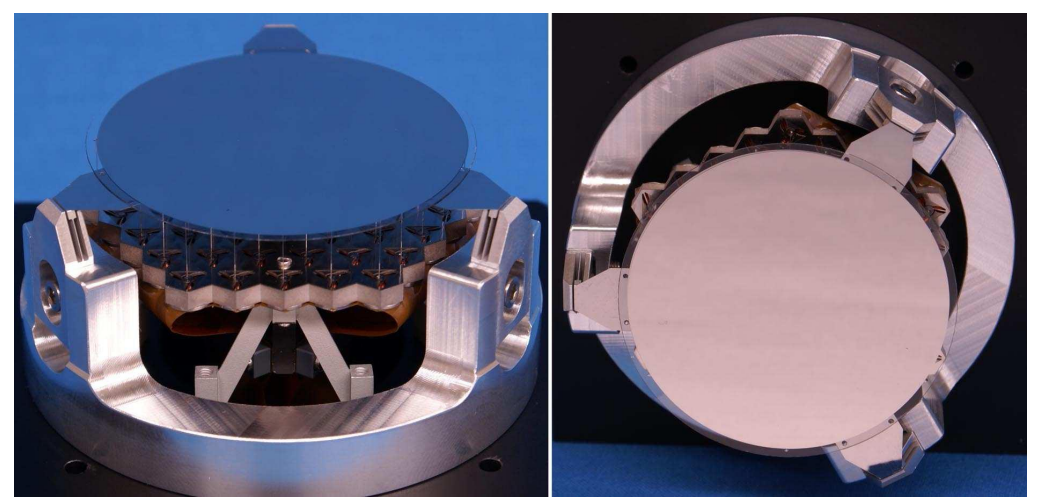

Figure 14. Pictures of the 61-actuator prototype
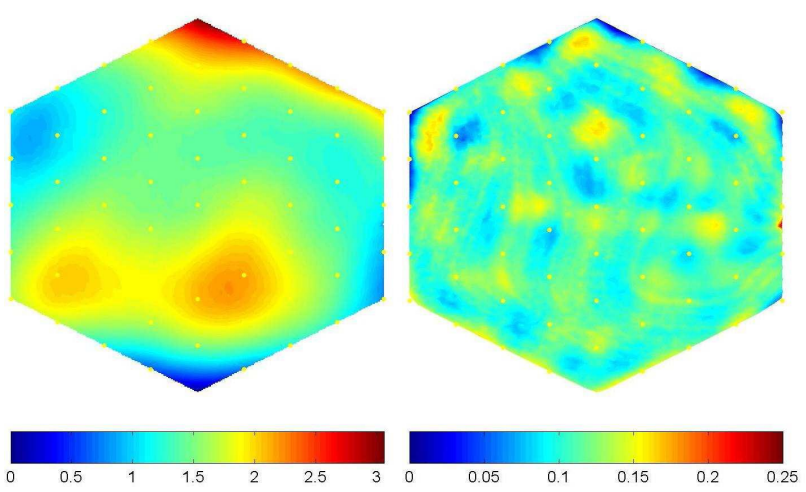

Figure 15. Interferogram of the 61 rod dummy mirror as before (left) and after correction with the theoretical influence functions (right).
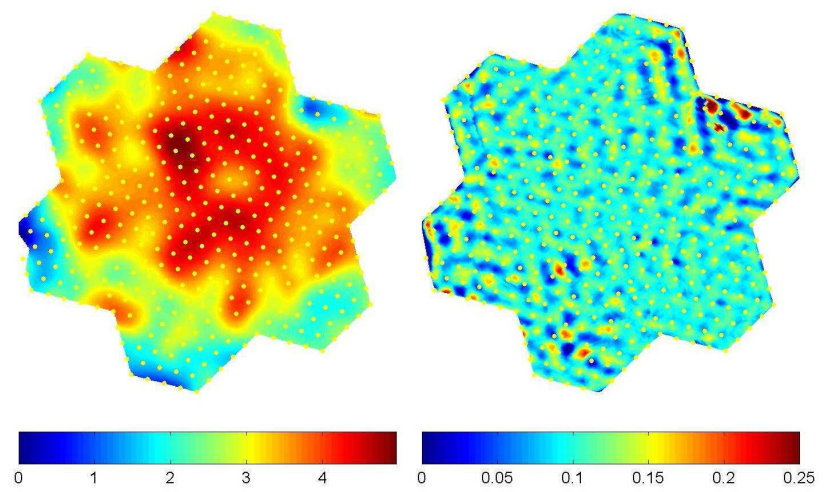

Figure 16. Interferogram of the 427 rod dummy mirror as before (left) and after correction with the theoretical influence functions (right).

\subsection{The actuator}

After actuator grid assembly, measurements were performed on the single actuators without the presence of the reflective membrane. From these measurements, the transfer functions $h / U_{C_{l}}$ and $h / I_{R_{a}}$ between both the voltage $U_{C_{l}}$ and the actuator current $I_{R_{a}}$ and the actuator position $h(t)$ were estimated. For the current input case, a SigLab ${ }^{\mathrm{TM}}$ system has been used to generate white noise with a bandwidth of $22.5 \mathrm{kHz}$, that was connected to a current amplifier. The actuator position $h(t)$ was measured using a laser-vibrometer connected to the SigLab unit. An empirical transfer function estimate was obtained from the input-output data using the Welch averaging method. This is scaled by $R_{a}$ for comparison with the voltage input case and plotted in figure 11 .

For the voltage input case, the dedicated electronics described in this report were used. A Matlab ${ }^{\mathrm{TM}}$ XPC realtime computer was used to generate a white noise excitation signal and send with an update rate of $5 \mathrm{kHz}$ over the serial communication to the driver electronics. The voltage over the actuator coil and the laser-vibrometer output were logged by the XPC target using a NI-6025E I/O card. The transfer function estimated from these two signals is also plotted in figure 11. The shape of the Bode plots is as expected, but the resonance frequency of $1830 \mathrm{~Hz}$ is slightly higher than the $\sqrt{c_{a} / m_{a}} / 2 / \pi \approx 1630 \mathrm{~Hz}$ expected from the model.

\subsection{Influence functions and flattening}

The DM influence functions were measured using a Wyko 400 interferometer with the Intelliwave wavefront reconstruction software. The influence function of each actuator was estimated based on measurements for five different setpoint values. Since the interferometer cannot measure the global average (i.e. piston), this term is not included in the estimates. For two actuators these are plotted in figure 17 together with the ones expected from the model derived in the previous section. It matches the measurements well, with only slight gain variations 

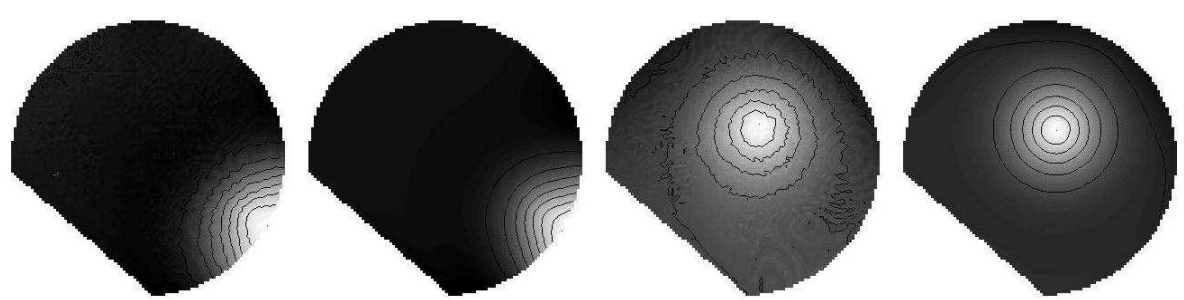

Figure 17. Influence functions of two actuators as estimated from interferometer images (1 and 3$)$ and as modeled (2 and $4)$.

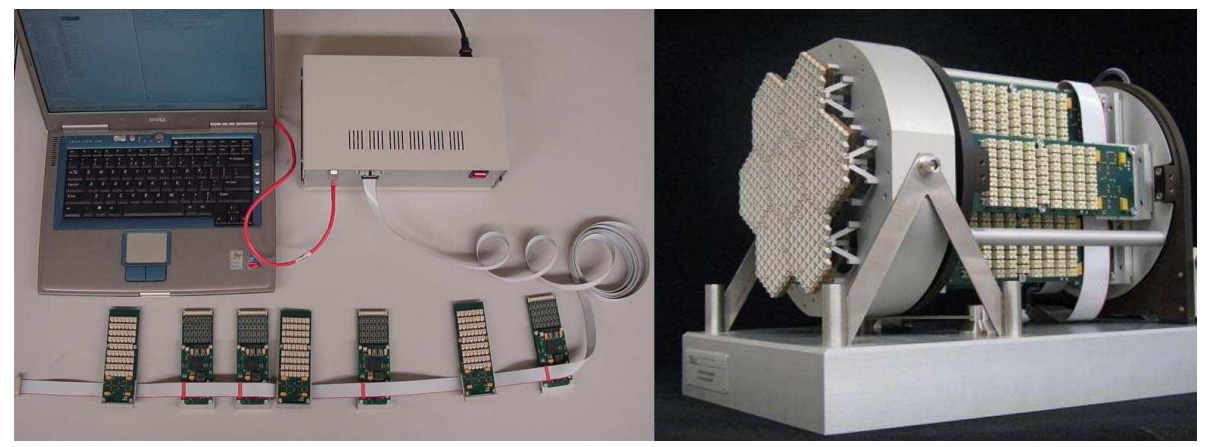

Figure 18. The Ethernet-LVDS bridge and the multidrop cable that connects 7 PCB's with 61 actuator drivers each (Left). The 7427 actuator mirror prototype in its final assembly stage. As a last step the membrane is placed in front of the 7 actuator grids.(right)

that were to be expected.

From the assembling a slight initial non-flatness of the reflective membrane is expected and needs to be corrected. This is budgeted in the order of a few $\mu \mathrm{m}$, requiring a maximum of $10 \%$ of the available actuator force. However, in the first prototype the assembly procedure had several shortcomings, leading to a larger initial non-flatness of $5 \mu \mathrm{m}$ peak-to-valley (PTV) or $0.75 \mu \mathrm{m}$ root-mean-square (RMS). An iterative flattening procedure that exploited the measured influence matrix was used to find actuator signals that minimize the residual nonflatness in a least squares sense. Using this procedure, the non-flatness of the central area containing 19 actuators was reduced to $12 \mathrm{~nm}$ RMS, showing no print-through of the glued actuator rods.

\subsection{The electronics}

The communication latency can be split into several parts. First, the data has to be sent over a 100Mbit/s ethernet connection. For 427 actuators on seven modules this takes approximately $75 \mu \mathrm{s}$. Then the communication bridge takes $85 \mu$ s of processing time before transmitting over the LVDS connection, which takes $30 \mu$ s per module. The first actuator thus receives its setpoint after $75+85+30=190 \mu \mathrm{s}$ and the last after $75+85+7 \cdot 30=370 \mu \mathrm{s}$. The maximum update rate is limited by the speed of the LVDS connection to $1 /\left(7 \cdot 30 \cdot 10^{-6}\right)=4760 \mathrm{~Hz}$. This is much higher than the foreseen sampling frequency of the control system, but when 32 modules are connected the $32 \cdot 61=1952$ actuators can be updated at approximately $1 \mathrm{kHz}$. Alternatively, the high sampling frequency can be used for model identification and validation purposes. Although this higher frequency reduces the oversampling ratio of the PWM signal, this remains above 12 and should be sufficient.

Although the PWM principle to generate the actuator voltages is very power efficient, the FPGA's that generate these signals currently dissipate $600 \mathrm{~mW}$ for 61 actuators. Therefore, asynchronous implementations are being investigated that are expected to drastically reduce power consumption.

\section{NEXT STEPS}

Currently the 427 actuator DM prototype is in its final assembly stage. All 7 actuator grids including driver and communication electronics are functional (figure 18). The test assembly with 427 rods to a $150 \mathrm{~mm}$ diameter 
$100 \mu \mathrm{m}$ thick Pyrex membrane and dummy base was successful and the final step - assembling the membrane to the actuator grids - are expected to be complete mid June 2008. Measurements of the initial optical quality, influence function measurements and initial flattening as well as closed loop measurements on the AO breadboard are foreseen.

\section{CONCLUSIONS}

A new DM design for future, large AO-systems is presented that consists of a thin, continuous membrane on which push-pull actuators impose out-of-plane displacements. Each actuator has $\pm 10 \mu \mathrm{m}$ stroke, nanometer resolution and only mW's heat dissipation. A fully functioning prototype has been made, including the required driving and communication electronics. Test measurements were performed, including influence functions and transfer functions of single actuators. They correspond well to the derived linear models. First measurements for a dummy mirror supported by 427 rods representing the actuators are presented, indicating an initial nonflatness that is compensated by less than than $10 \%$ of the available actuator force. The full assembly procedure of the 427 actuator prototype will be complete around mid June 2008.

\section{ACKNOWLEDGMENTS}

The presented work is partially funded by Senter Novem, the Dutch Innovative Research Project (IOP) Precision Technology.

\section{REFERENCES}

[1] Hamelinck, R., Rosielle, N., Steinbuch, M., Ellenbroek, R., Verhaegen, M., and Doelman, N., "Actuator tests for a large deformable membrane mirror," in [Proceedings of the SPIE conference on astronomical telescopes and instrumentation- advances in adaptive optics], 627225, SPIE (May 2006).

[2] Ellenbroek, R., Verhaegen, M., Hamelinck, R., Doelman, N., Steinbuch, M., and Rosielle, N., "Distributed control in adaptive optics - deformable mirror and turbulence modeling," in [Proceedings of the SPIE conference on astronomical telescopes and instrumentation], 62723K, SPIE (May 2006).

[3] Hamelinck, R., Rosielle, N., Steinbuch, M., Ellenbroek, R., Verhaegen, M., and Doelman, N., "Test results of an adaptive deformable mirror for future large telescopes," in [Conference Proceedings of the European Society for Precision Engineering and Nanotechnology], (2008).

[4] Loktev, M., Monteiro, D. W., and Vdovin, G., "Comparison study of the performance of piston, thin plate and membrane mirrorsfor correction of turbulence-induced phase distortions," Optics Communications 192, 91-99 (May 2001).

[5] Timoshenko, S. and Woinowsky-Krieger, S., [Theory of plates and shells], McGraw-Hill, Auckland, second edition ed. (1989).

[6] Sims, J. R., Durney, A. N., and Smith, C. C., "Design of mechatronic systems with aliased plant modes," IEEE/ASME transactions on mechatronics 3, 144-149 (1998).

[7] Hansen, D. S., Digital controller design for alised sampled-data systems, PhD thesis, Brigham-Young university, department of mechanical engineering (August 2000).

[8] Hinnen, K. J., Data-Driven Optimal Control for Adaptive Optics, phd dissertation, Delft University of Technology (January 2007).

[9] Franklin, G. F., Powell, J., and Emami-Naeini, A., [Feedback control of dynamic systems], Addison-Wesley, 3rd ed. (1994). 